(C) 2018 IEEE. Personal use of this material is permitted. Permission from IEEE must be obtained for all other uses, in any current or future media, including reprinting/republishing this material for advertising or promotional purposes, creating new collective works, for resale or redistribution to servers or lists, or reuse of any copyrighted component of this work in other works. 


\section{Application-Oriented Robust Design Optimization Method for Batch Production of Permanent Magnet Motors}

\begin{abstract}
From the perspective of industrial production, the design and optimization of electrical machines are application oriented, including maximizing production quality and minimizing production cost in terms of different manufacturing conditions. To achieve these goals, this work presents an efficient application-oriented robust design optimization method for permanent magnet (PM) motors. The method consists of two main contributions. The first one is the development of an overall optimization strategy including qualitative and quantitative analyses to provide possible options for an application. Multi-physics analysis, uncertainty analysis, production cost and optimization models need to be investigated. The second one proposes a multilevel optimization method for the high-dimensional robust design problem of each option. To illustrate the advantages of the proposed method, PM motors with soft magnetic composite cores are investigated for domestic applications. The design optimization is conducted in terms of three motor options and three batch production volumes for both conventional deterministic and robust approaches, and it consists of eighteen high-dimensional multi-physics optimization problems in total. Main optimization results are presented and discussed. Experimental and simulation results are presented to validate the effectiveness of the proposed models and methods.
\end{abstract}

Index Terms - Application-oriented design optimization, permanent magnet motors, production cost, robust optimization.

\section{INTRODUCTION}

$\mathrm{E}$ LECTRICAL machines are the heart in many modern appliances, as well as industry equipment and systems. In a global market and in the context of sustainability, they must fulfill various requirements physically and technologically. To satisfy these requirements, optimization is of great significance for electrical machine design, and many optimization methods have been developed. Optimization methods mainly include (i) direct optimization of analysis models, such as analytical model, magnetic equivalent circuit model and finite element model (FEM), and (ii) indirect optimization of approximation models (surrogate models of FEM), by using different kinds of optimization algorithms including intelligent algorithms. Some popular intelligent algorithms are genetic algorithm (GA), differential evolution algorithm (DEA) and particle swarm optimization (PSO) algorithm. Surrogate models mainly include the parametric models, such as response surface model (RSM) and radial basis function (RBF) model, semi-parametric model, e.g., Kriging model, and non-parametric models, such as artificial neural network model and support vector machine model [1]-[8].
On the other hand, with the fast development of CAD/CAE software, new and advanced materials, flexible machinery technology and intelligent optimization methods, it is possible to design and manufacture a motor to meet the special requirements of an application. Thus, the design optimization is application oriented. This approach is of great significance for both designers and manufacturers. However, there are three main challenges for the efficient implementation of this approach to electrical machines, which can be illustrated in terms of the following three perspectives.

Firstly, from the perspective of design, to obtain a good motor to meet the requirements, the most possible options and/or combinations including motor types, topologies, materials and dimensions should be investigated in the design optimization process. This idea is not novel but the key is the efficiency, i.e., how to handle the huge computation burden required in the implementation as many options should be optimized and each one is usually a high-dimensional multi-physics problem [1], [9], [10].

Secondly, from the point of view of manufacturing, the final quality of a motor in production depends highly on the manufacturing technology employed and can be greatly affected by essential manufacturing tolerances and unavoidable material and assembling uncertainties [11]-[16]. Table I lists some general factors for permanent magnet (PM) motors. The effects of manufacturing tolerances and assembly variations were studied in reducing the cogging torque and harmonics of PM motors recently [13], [16].

Fig. 1 shows an example to address the issue why manufacturing quality is required to be investigated in the design optimization stage of motors. As shown, a multi-objective optimization is proposed aiming for low material cost, high output power for a given volume (power density), and high manufacturing quality in terms of sigma level of a PM transverse flux motor. The sigma $(\sigma)$ rating has been commonly used by industry to describe the maturity of a manufacturing process to indicate its yield or the percentage of defect-free products it creates. For the long-term quality control, a $6 \sigma$ process is actually one in which $99.99966 \%$ of all opportunities are expected to be free of defects, i.e. 3.4 defects per million opportunities (DPMO). As a comparison, $4 \sigma$ is equivalent to 6,200 DPMO [1], [17].

The motor is firstly optimized with the material cost and output power as the objectives, and the optimal Pareto solutions are plotted as the black circles on the 2D plane of material cost versus output power. The motor is then optimized again with the manufacturing quality as the third objective with the investigation of manufacturing tolerances of PM and winding. 
The resultant optimal Pareto solutions are plotted as red squares in the 3D space with the green points as the projections in the 2D plane of material cost versus manufacturing quality. As shown, the optimal Pareto solutions can be divided into two subsets: low and high-quality optimums. Aggressive designs of high output power (above $750 \mathrm{~W}$ in this case) will result in low manufacturing quality with $4 \sigma$ at the best. In most cases, the designs of moderate material cost and output power (around AUD32 and 730-750 $\mathrm{W}$ in this case) would be chosen. As shown by the cylinder in Fig. 1, some of the moderate designs have low manufacturing quality (less than $4 \sigma$ ). Only those on the top end of cylinder meet the target of all three objectives [17]. Thus, an aggressively optimized design obtained by the deterministic optimization (conventional method, without consideration of manufacturing uncertainties) may be difficult to mass produce and it may end up with high manufacturing cost and/or high rejection rates. Therefore, the robust approach is necessary for design optimization of motors from the perspective of industrial applications [1], [17].

TABLE I

MANUFACTURING AND MATERIAL VARIATIONS IN PM MOTORS

\begin{tabular}{lll}
\hline \hline Description & Ideal & Variation \\
\hline Magnet dimension & Nominal & Nominal $\pm \Delta$ Tol \\
Magnet strength & Nominal & Nominal $\pm 5 \%$ \\
Magnet disposition & $0 \mathrm{deg}$ & $1.0 \mathrm{deg}$ \\
Magnetization offset & $0 \mathrm{deg}$ & $1.0 \mathrm{deg}$ \\
Skew error & Nominal & Nominal $+0.67 \mathrm{deg}$ \\
Copper diameter & Nominal & Nominal $\pm \Delta T o l$ \\
Eccentricity & $0 \mathrm{~mm}$ & $0.35 \mathrm{~mm}$ \\
\hline \hline
\end{tabular}

$\Delta \overline{\Delta T o l \text { stands for manufacturing tolerance }}$

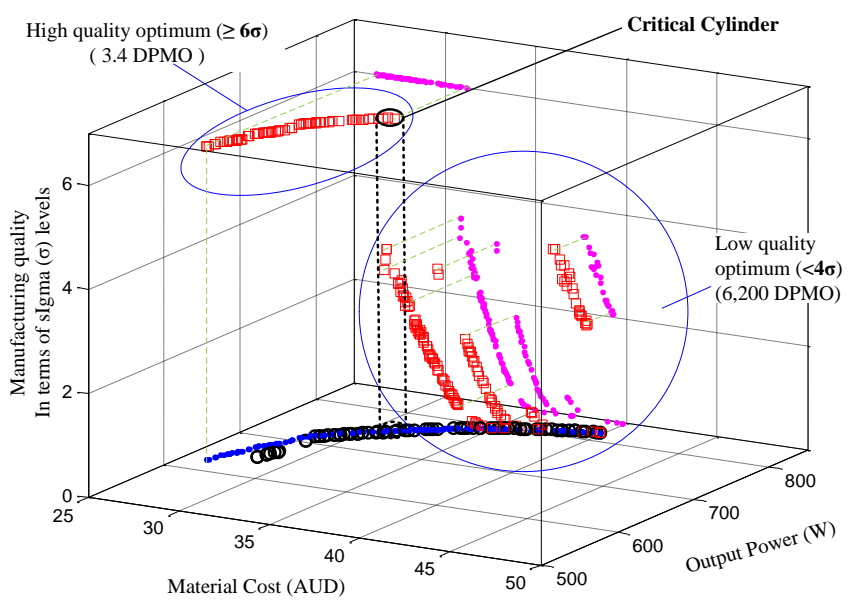

Fig. 1. Illustration of design optimization for $6 \sigma$ manufacturing quality.

Thirdly, from the perspective of market, the production cost is a critical issue for successful design and application of an electrical machine. The manufacturing or production cost estimation has long been a difficult task for electric machine designers. For the practical production, different manufacturers have different manufacturing facilities and production volumes, which would result in different design optimums [18]. Therefore, the robust design optimization should be implemented separately in terms of production volumes/ manufacturers, and this is a new problem. Thus, not only the motor performance but also the production cost and quality are very important for both manufacturers and customers.

To attempt these challenges, this work presents an effort to develop an application-oriented robust design optimization method for electrical machines with the focus on PM motors. It is organized as follows. Section II presents the proposed method. Section III describes an integrated product and process development model for estimation of production cost of PM motors. Section IV introduces the multilevel robust optimiz ation method. Section $\mathrm{V}$ presents an example study for design optimization of PM motors with soft magnetic composite (SMC) cores for domestic applications with detailed steps and results. Experimental and simulation results are provided in Section VI, followed by the conclusion section.

\section{APPLICATION-ORIENTED RobUSt DESIGN OPTIMIZATION METHOD FOR PM MOTORS}

Fig. 2 shows a framework for the proposed applicationoriented robust design optimization method for PM motors. It mainly includes the following five steps.

Step 1: Define the specifications or requirements of the designed motors in terms of specific applications, such as refrigerators and hybrid electric vehicles (HEVs), including the rated speed, output power and volume. Meanwhile, manufacturing quality target including reliability should be determined in this step.

Step 2: Determine the potential design options, such as motor types, topologies and materials. Even when the motor type is chosen, there could be various options.

The electric vehicles, either battery powered or plug-in HEVs, are good examples, which are attracting great attentions from governments and public around the world because of the worldwide energy sustainability and environment protection. To meet the challenging requirements, many kinds of PM motors have been investigated to improve the drive performance of EVs, such as interior PM motor and flux-switching PM machines (FSPMMs). Taking the FSPMM as a further investigation, many topologies can be selected as design options, such as radially and axially laminated steel sheets for the stator core, various winding configurations, and different combinations of stator/rotor poles [19]-[21].

Another example could be the PM-SMC motors. SMC material is a relatively new soft magnetic material that is composed of surface electrically insulated iron powder particles, which results in low eddy current loss, and magnetic and thermal isotropy, making PM-SMC motors good candidates for many applications [22]-[28]. In terms of domestic applications, such as compressor drives in refrigerators and air-conditioners, some popular design options are transverse flux machine (TFM), claw pole machine (CPM) and axial flux machine [1], [24], [27]-[30].

As discussed, different materials can be employed to design the stator cores of PM motors, such as silicon steel sheet and SMCs. For PMs, the rare-earth and ferrite magnets are two popular options. All these factors are directly related to the output performance and safe operation of the designed machines, such as torque and temperature rise.

Step 3: Establish initial design for each option. This design should consist of multi-disciplinary analysis model, production cost estimation model and robust analysis. This step also 
includes the determination of initial dimension and performance evaluation for each motor option, such as torque, efficiency and temperature rise. The analysis model can be analytical model or FEM.

It should be noted that the manufacturing method and production process must be investigated in this step. For example, by using the powder metallurgical technology, the PM motors with SMC cores can be manufactured in a convenient and economical way. By using this manufacturing method, motors with SMC cores have very low material waste (less than 5\%) during the manufacturing process. It also has the merits of the net shape, smooth surface and good tolerances for the electrical machines [26]. The robust analysis includes the determination of material variations and manufacturing tolerances or distribution parameters. Then its optimization model can be defined by using the design for six sigma (DFSS) technique.

Step 4: Develop a uniform optimization model for all options and optimize each option to acquire its optimal design parameters and performance by using the optimization methods, such as GA, PSO, and RSM and Kriging model. It should be noted that, multilevel optimization strategy can be employed here to improve the optimization efficiency for this kind of high-dimensional design problems [1], [31].

Step 5: Compare the optimal results of all options, and output the best one as the final optimal solution for that specific application.

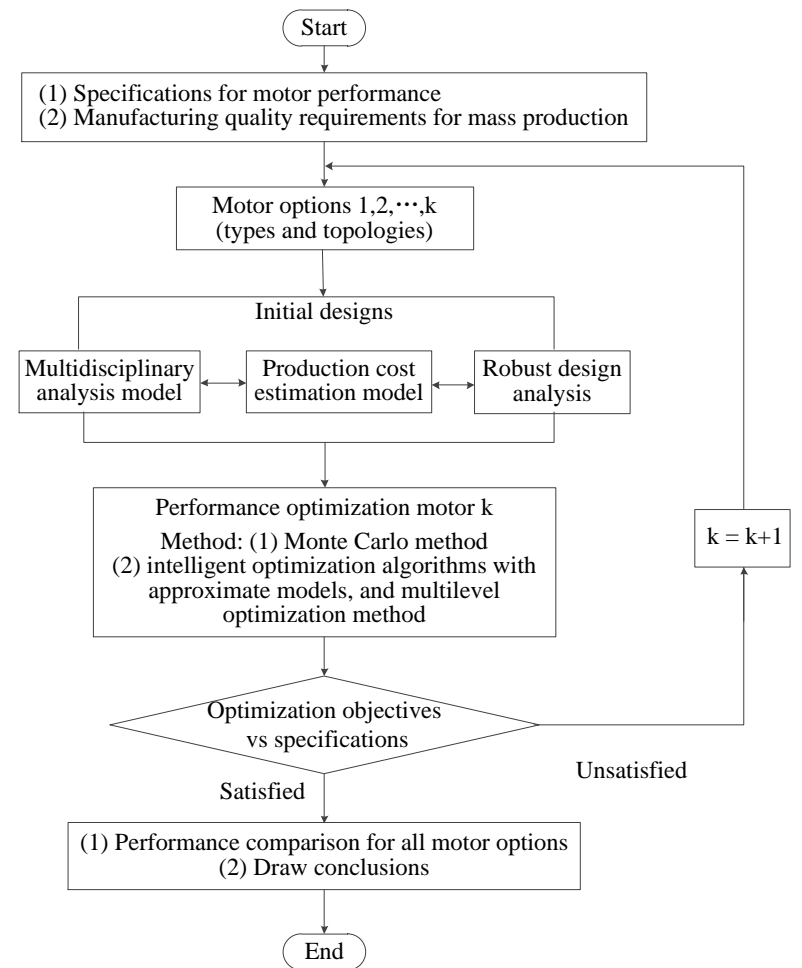

Fig. 2. Flowchart of application oriented robust design optimization method.

\section{INTEGRATED PRODUCT AND PROCESS DEVELOPMENT MODEL FOR PM MOTORS}

For different production volumes, the production cost of the same design may vary significantly, since it can be influenced by production technologies and management. Thus, it is hard to conclude that one optimal design is suitable for various production conditions. And it is of great significance if the production cost rather than material cost can be considered when handling with electrical machine design optimization. To achieve an estimation method for the production cost of PM motors, an integrated product and process development model is introduced in this section and will be employed in the following design optimization [18]. The proposed model mainly includes the following two aspects for PM motors.

\section{A. Determination of the process chain}

Generally, the process chain consists of housing, stacks/disks, rotor, stator, shaft and final assembly for production of PM motors. Each step in the chain may include several tasks, for example, winding and slot isolation are normal tasks for a stator construction. In some cases, PMs are placed in the stator (e.g., FSPMM), and then magnetizing the PMs is a critical task. For the final assembly, normal tasks are the adjustment, sensor placement and joining of rotor, stator and housing. Fig. 3 shows a typical process chain of PM-SMC motors. As shown, there are four major tasks in the stator step as the stator is made of SMC material and this kind of material has new manufacturing method. The manufacturing of shaft and housing is based on the industrially mastered standard procedures.

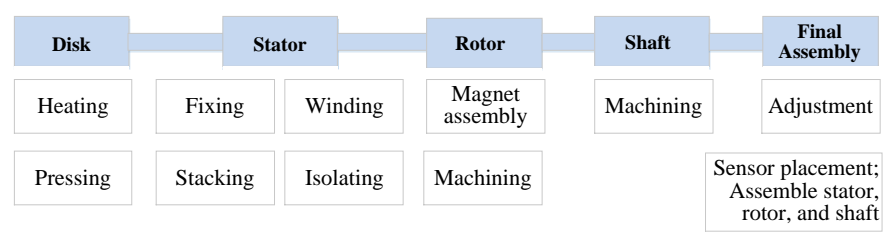

Fig. 3. Illustration of process chain for PM-SMC motor production.

\section{B. Production cost model/function}

Production cost is normally composed of fixed costs (such as building and depreciation for machines) and variable costs (such as wages, energy, material and maintenance). In general, small volumes imply low fixed costs (produced on machinery with low investment cost), and higher variable costs (higher share of manual work). For high volumes, the production costs can be decreased by using expensive machinery that allows automation and thus decreasing the variable costs. Hence, different technologies or machine sizes offer diverse production volumes [18], [32].

Theoretically, the production cost can be expressed as a function of production volume $(p v)$ and the design parameters $(\mathbf{x})$ including material and dimension. It mainly consists of five parts, machinery cost, material cost, buying-parts cost, capital cost and personnel cost. The material cost can be calculated by$$
C_{\text {matc }}(p v, \mathbf{x})=M u(\mathbf{x}) \cdot M p(p v)-M u(\mathbf{x}) \cdot \operatorname{sr}(\mathbf{x}) \cdot s p(p v)
$$
where $M u$ is the material usage, $M p$ is the material price, $s r$ is the scrap portion, and $s p$ the price for scrap part. For the buying parts, its cost can be estimated by

$$
C_{\text {partc }}(p v, \mathbf{x})=\sum_{i=1}^{n p} P_{p}(p v, \mathbf{x}) N_{p}
$$

where $P_{p}$ and $N_{p}$ are the price and number of the buying parts. Estimation models/methods of other parts are available in some research works or industrial projects/reports [32]. 


\section{Multilevel Robust Design Optimization MethoD}

A typical optimization model with respect to an objective $f(\mathbf{x})$ and $m$ constraints $g(\mathbf{x})$ has the form as

$$
\begin{array}{ll}
\min : & f(\mathbf{x}) \\
\text { s.t. } & g_{i}(\mathbf{x}) \leq 0, i=1, \ldots, m, \\
& \mathbf{x}_{l} \leq \mathbf{x} \leq \mathbf{x}_{u}
\end{array}
$$

where $\mathbf{x}_{l}$ and $\mathbf{x}_{u}$ are the boundaries of the design parameter $\mathbf{x}$.

This model can be taken as a deterministic model as $\mathbf{x}$ is deterministic and does not contain any manufacturing and material uncertainties as shown in Table I. To solve this problem, (3) can be converted into a robust design optimization model based on a technique called design for Six-Sigma (DFSS). In the DFSS, all design parameters (including material and dimension) are assumed to follow normal distributions with different means $(\mu)$ and standard deviations $(\sigma)$, thus to reflect the manufacturing and material variations. From this prospective, the robust model has the from as

$$
\begin{aligned}
\min : & F\left[\mu_{f}(\mathbf{x}), \sigma_{f}(\mathbf{x})\right] \\
\text { s.t. : } & g\left[\mu_{f}(\mathbf{x}), \sigma_{f}(\mathbf{x})\right] \leq 0, i=1, \ldots, m \\
& \mathbf{x}_{l}+n \sigma_{\mathbf{x}} \leq \mu_{\mathbf{x}} \leq \mathbf{x}_{u}-n \sigma_{\mathbf{x}} \\
& \mathrm{LSL} \leq \mu_{f} \pm n \sigma_{f} \leq \mathrm{USL}
\end{aligned}
$$

where LSL and USL are the specification limits, and $n$ is the sigma level, which is equivalent to a probability in terms of a standard normal distribution as shown in Table II. For example, $4 \sigma$ is equivalent to a reliability of $99.9937 \%$, or 63 DPMO. It seems good enough for manufacturing. However, this is true only in terms of statistics or short-term quality control. For the long-term quality control, an $1.5 \sigma$ shift in the mean has been observed by many enterprises. As a result, $4 \sigma$ is equivalent to 6,200 DPMO, which is quite large. Thus, $6 \sigma$ has been adopted by many companies nowadays as it yields 3.4 DPMO only.

Meanwhile, to estimate $\mu$ and $\sigma$ in (4), Monte Carlo analysis (MCA) is usually required. MCA is a classic statistical analysis technique for characterizing the uncertainty based on repeated random sampling. The sample size is usually big, for example, 10,000 , which will result in huge computation burden in the implementation of (4).

In general, there are two strategies for the optimization of the model (4), single-level and multilevel methods. Single-level method optimizes all parameters at the same time, resulting in huge computation cost. The multilevel robust optimization method as shown in Fig. 4 is introduced to overcome this problem. As shown, it consists of four main steps.

Step 1: Determination of the sigma level in terms of design requirements and available manufacturing conditions.

Step 2: Dividing the initial space into 3 subspaces (X1, X2 and X3) according to the sensitivity of parameters.

Step 3: Optimizing subspaces sequentially. The parameters in the other two subspaces are fixed when the optimization is applied to one subspace. In the implementation, as the dimension of each subspace is much smaller than that of the initial design space, the approximation model, e.g., Kriging model, can be employed to improve the optimization efficiency [33]. This model can be used for MCA estimation. Therefore, the computation burden can be decreased greatly. The inputs for each level include fixed and optimization parameters, and their uncertainties. The outputs of each level mainly include motor performances and MCA data. Detailed steps can be found in the left hand side of Fig. 4.

Step 4: Updating process. If the optimized results do not satisfy requirements, update $\mathrm{X} 2$ and $\mathrm{X} 3$ and go to step 3 again until all requirements are met.

TABLE II

MANUFACTURING DPMO IN TERMS OF SIGMA LEVEL

\begin{tabular}{cccc}
\hline $\begin{array}{c}\text { Sigma } \\
\text { level }\end{array}$ & Percentage & $\begin{array}{c}\text { DPMO } \\
\text { (short term) }\end{array}$ & $\begin{array}{c}\text { DPMO } \\
\text { (long term) }\end{array}$ \\
\hline $1 \sigma$ & 68.26 & 317,400 & 697,700 \\
$2 \sigma$ & 95.46 & 45,400 & 308,733 \\
$3 \sigma$ & 99.73 & 2,700 & 66,803 \\
$4 \sigma$ & 99.9937 & 63 & 6,200 \\
$5 \sigma$ & 99.999943 & 0.57 & 233 \\
$6 \sigma$ & 99.9999998 & 0.002 & 3.4 \\
\hline \hline
\end{tabular}

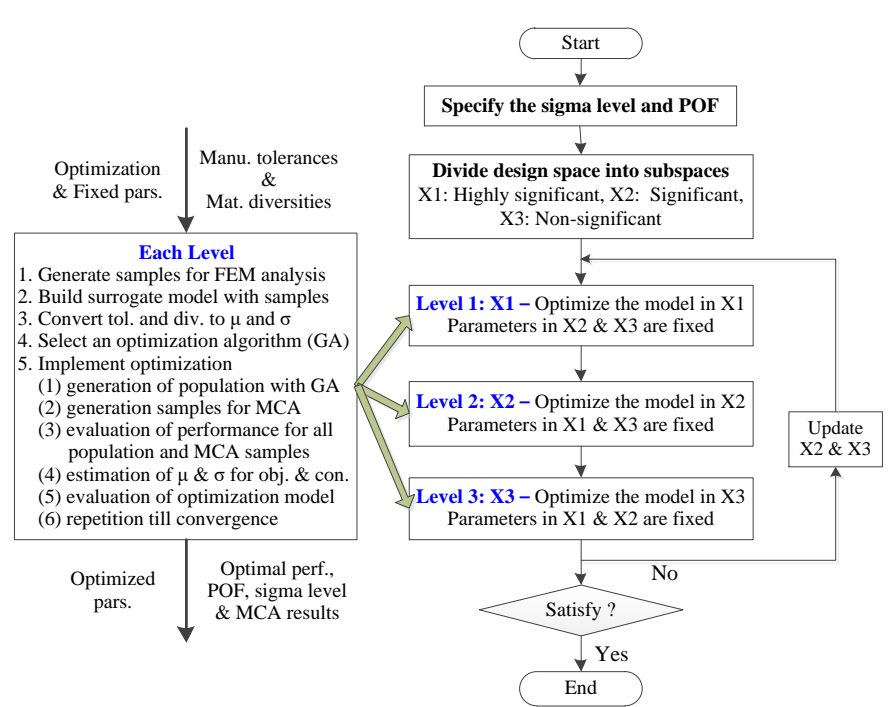

Fig. 4. Flowchart of the multilevel robust design optimization method.

\section{AN APPLICATION EXAMPLE FOR PM-SMC MOTORS}

\section{A. Application requirements/specifications}

In this example, a PM-SMC motor is designed for a refrigerator with specifications as listed in Table III.

\section{B. Motor options}

Three motor topologies, CPM, TFM and axial flux machine have been widely discussed in the previous work. From a recent comparative study, the CPM and TFM have advantages over the axial flux one [27]. Thus, CPM and TFM will be investigated in this work. Table IV lists some design and performance parameters for CPM and TFM based on two prototypes developed in the previous work [1], [15]. As shown, CPM is better than TFM in terms of flux concentrating ability and PM usage.

Moreover, the good flux concentrating ability of the CPM offers the possibility for replacing the rare-earth PM by ferrite PM. Compared with rare earth PM, ferrite PM usually has lower magnetic energy product and density, but its cost is much cheaper than the rare earth PM. Therefore, it is worthwhile to investigate the feasibility of ferrite CPM for the proposed application under its specific requirement. In total, three motor 
options are determined for the given application, and they are CPM with rare earth PM, CPM with ferrite PM and TFM with rare earth PM. Fig. 5 shows the 3D design structure and structural parameters for the CPM, which applies for both rare earth PM and ferrite PM options. Fig. 6 shows the 3D design structure and structural parameters for the TFM.

TABLE III

SPECIFICATIONS OF THE TARGET MOTOR

\begin{tabular}{cccccc}
\hline \hline Requirement & Unit & Value & Requirement & Unit & Value \\
\hline Rated speed & rpm & 1800 & Outer radius & $\mathrm{mm}$ & 55 \\
Rated power & $\mathrm{W}$ & 675 & Axial length & $\mathrm{mm}$ & 100 \\
Rated efficiency & $\%$ & $\geq 80$ & Supply voltage & $\mathrm{V}$ & 230 \\
\hline \hline
\end{tabular}

TABLE IV

SeVeral Design and Performance Parameters

\begin{tabular}{cccc}
\hline \hline Parameter & Unit & CPM & TFM \\
\hline Effective stator axial length & $\mathrm{mm}$ & 93 & 93 \\
Rotor outer radius & $\mathrm{mm}$ & 47 & 47 \\
Turns of coil & - & 75 & 125 \\
PM piece & - & 60 & 120 \\
Motor back EMF constant & $\mathrm{V} / \mathrm{rpm}$ & 0.0271 & 0.0252 \\
Flux of one turn & $\mathrm{mWb}$ & 0.488 & 0.272 \\
Rated power & $\mathrm{W}$ & 500 & 640 \\
\hline \hline
\end{tabular}

C. Initial designs with multi-physics analysis model, production model and robust analysis

(a) Multi-physics analysis model

Multi-physics analysis model of PM-SMC motors mainly includes electromagnetic, thermal and modal analyses, which has been discussed in the previous work. Fig. 7 shows an illustration of the magnetic field for the TFM. Due to the 3D flux nature, 3D FEM is required for the analysis and optimization of these SMC motors. Other models related to the thermal and model analyses can be found in [1]. Fig. 8 shows a 3D thermal network model for the middle stack of the studied PM-SMC TFM. $R_{s y}, R_{s d}, R_{c u}, R_{s t}, R_{g}, R_{p m}, R_{r t}, R_{r y}$ and $R_{s f}$ are equivalent thermal resistances of stator yoke, stator side disk, coils, stator teeth, air gap, PMs, rotor radial part, rotor axial part and shaft, respectively. The heat sources in this model include the stator core loss $\left(P_{F e s 1}\right)$, rotor core loss $\left(P_{F e r 1}\right)$, copper loss $\left(P_{c u 1}\right)$, and mechanical loss $\left(P_{m e c h 1}\right)[30]$.

(b) Production cost estimation model

As discussed above, the production cost is determined by the design parameters and the manufacturing processes under the production condition for specific production volume. The process chain of PM-SMC motors can be derived with the structure as shown in Fig. 2. For example, Fig. 9 shows the process chain for the TFM with rare earth PM. To produce this motor, special considerations should be given to the SMC stator as the molding technique is required. Three cost parts are investigated in this work for PM-SMC motors, which are the machinery, material and buying-parts costs. Based on previous experience, SMC core is critical for the motor performance and production cost as it can be manufactured by molding technology instead of lamination stacking. Thus, special efforts are provided for SMC core in this part.
Fig. 10 shows the manufacturing cost and productivity for SMC cores. As shown, the cost is directly proportional to the press size (press force) while the productivity is inversely proportional to that. For example, an 100-ton press can deliver 500 SMC core disks per hour with cost AUD100 (AUD $0.2 /$ disk); while a 500-ton press can only produce 100 core disks per hour with cost AUD500 (AUD5/disk). This is a big difference in mass production. Meanwhile, the press size with the design parameters also determines the core density, which influences the B-H characteristic (see Fig. 13) as well as motor performance. For the other parts of SMC motor, the manufacturing process is normal. Fig. 11 shows the material price and assembly costs in terms of three production volumes, where SV, MV and LV stand for small volume $(3,000$ motors/year), medium volume (30,000 motors/year), and large volume (150,000 motors/year), respectively. These data will be used in the following optimization.

(c) Robust analysis

For the PM-SMC motors, the robust analysis includes the variation analysis of both structural and material parameters. SMC core density and PM property and their variations affect the performance of the PM-SMC motors significantly. As examples, Fig. 12 shows an example of the pressed SMC core disk by a mold. Fig. 13(a) depicts the measured core densities of $18 \mathrm{SMC}$ cores used for heat treatment analysis generated by orthogonal experimental design. Obviously, there are big variations, which will result in big differences in their B-H curves according to the relationship between core density and magnetic characteristics as shown in Fig. 13(b).

For PMs, the manufacturing quality of PM is crucial to the performance of PM motors [12]-[14]. Among several PM property parameters, the remanent flux density $\left(B_{r}\right)$ and dimension affect the motor performance significantly. Several batches of PMs in terms of different rates (such as N30M, $\mathrm{N} 38 \mathrm{M}$ and N50M) have been measured in the experiments. As an example, Fig. 14 shows the measured distribution of $B_{r}$ and thickness (magnetization direction) of more than 100 pieces of N38M randomly selected from a batch. Big variations can be found for both cases, which will result in big performance variations. The obtained means and standard deviations will be employed to develop the robust optimization model.

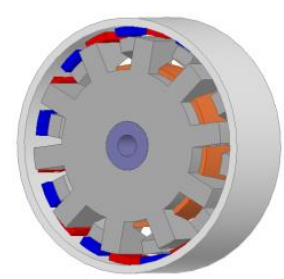

(a)

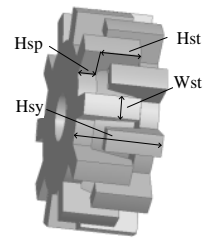

(c)

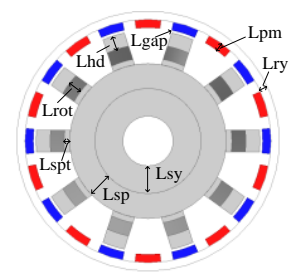

(b)

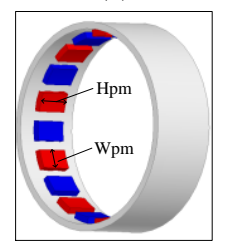

(d)
Fig. 5. 3D design structure and parameters of the CPM. 


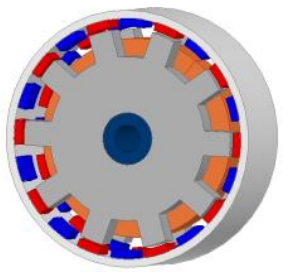

(a)

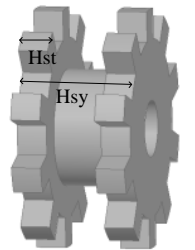

(c)

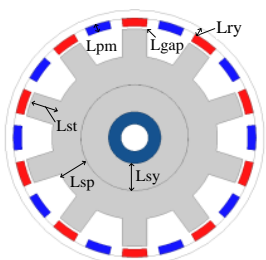

(b)

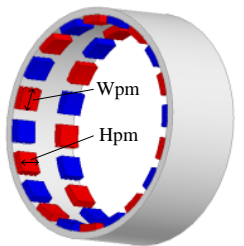

(d)
Fig. 6. 3D design structure and parameters of the TFM
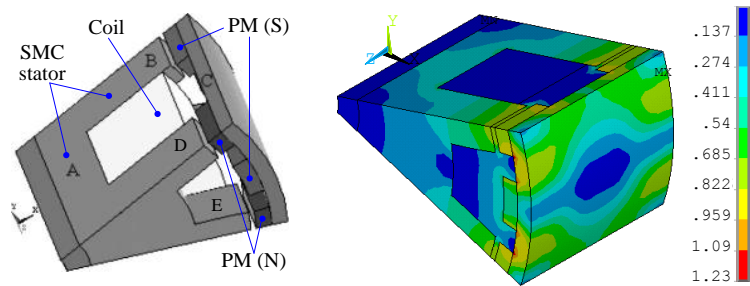

Fig. 7. FEM analysis region (left) and magnetic field distribution (Unit: T)

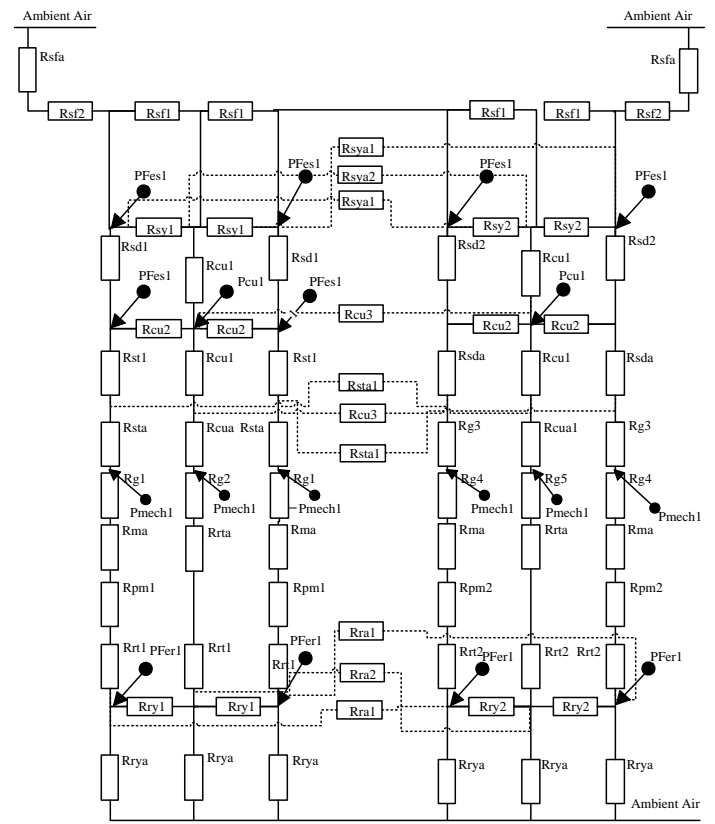

Fig. 8. 3D thermal network model of the PM TFM with SMC core.

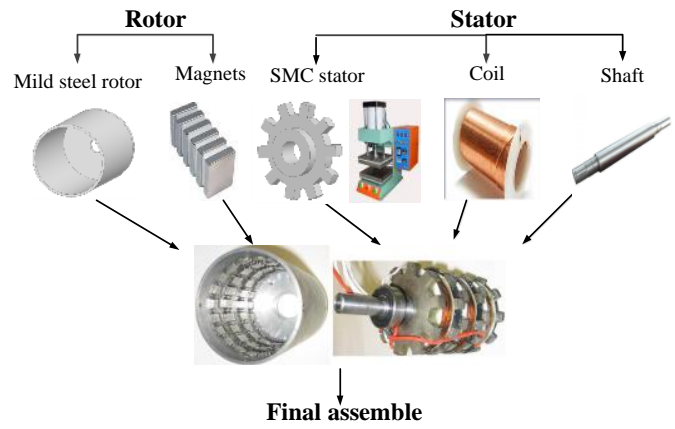

Fig. 9. Process chain of PM TFM with SMC.

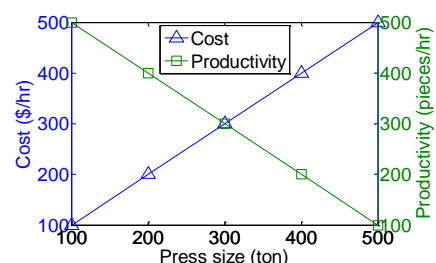

Fig. 10. Manufacturing cost and productivity for SMC cores.
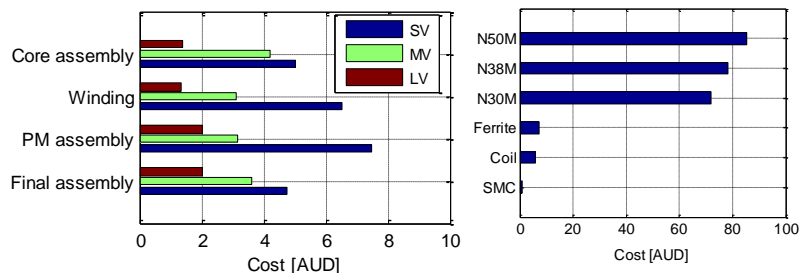

Fig. 11. Assembly (left) and material (right) cost for SMC motors.
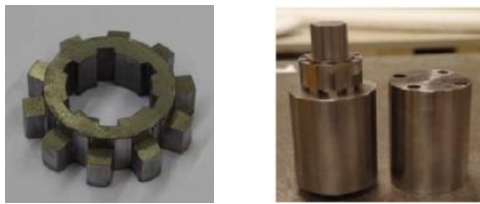

Fig. 12. A pressed SMC core disk (left) and the employed mold (right).

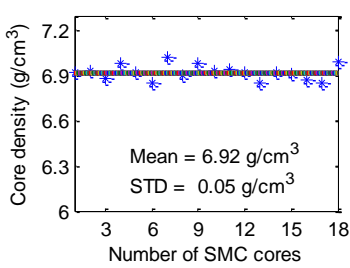

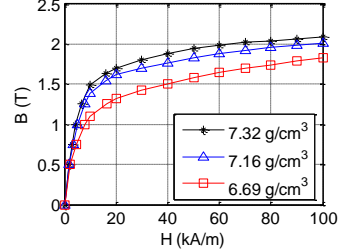

(b)
Fig. 13. (a) SMC core density variation, (b) B-H curves for cores.
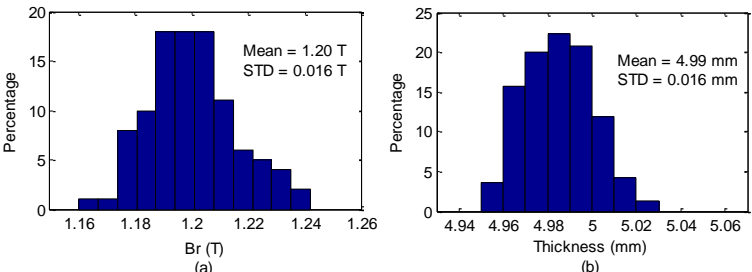

Fig. 14. Measured remanence and thickness distributions of a batch of PMs

\section{Optimization models and methods}

The optimization objectives are to minimize the product cost and maximize the efficiency $(\eta)$, while keeping the basic requirement. Sixteen parameters as listed in Table V for CPM (14 parameters for TFM) are included in the optimization. The deterministic optimization model can be defined as

$$
\begin{array}{ll}
\min : & f(\mathbf{x})=\text { Cost } / C_{\text {initial }}+\eta_{\text {initial }} / \eta \\
\text { s.t. } & g_{1}(\mathrm{x})=675-P_{\text {out }} \leq 0, g_{2}(\mathrm{x})=0.8-\eta<0 \\
g_{3}(\mathrm{x}) & =s f-0.7<0, g_{4}(\mathrm{x})=V_{m}<V_{\text {spec }} \\
g_{5}(\mathrm{x}) & =T_{\text {coil }}-75<0
\end{array}
$$

where subscript initial stands for the values obtained from the initial design as shown in Table V. $P_{\text {out }}$ and $s f$ are the output power and slot fill factor, respectively, $V_{m}$ and $V_{s p e c}$ are the optimized volume and that given in the specification. $T_{\text {coil }}$ is temperature rise in the winding, and 3D thermal network model is used for its calculation. Then for the robust optimization, the model has the form 
TABLE V INITIAL DESIGNS OF THE TWO CPMS WITH NDFEB AND FERRITE PMS

\begin{tabular}{ccccc}
\hline \hline Par. & Description & Unit & NdFeB & Ferrite \\
\hline$L_{s y}$ & Length of stator yoke & $\mathrm{mm}$ & 12 & 13 \\
$L_{s p}$ & Length of stator plate & $\mathrm{mm}$ & 10 & 13 \\
$L_{r o t}$ & Length of tooth root & $\mathrm{mm}$ & 5 & 5 \\
$L_{h d}$ & Length of tooth head & $\mathrm{mm}$ & 5 & 5 \\
$L_{g a p}$ & Length of air gap & $\mathrm{mm}$ & 1 & 1 \\
$L_{p m}$ & Length of PM & $\mathrm{mm}$ & 3 & 6 \\
$W_{s t}$ & Tooth circumferential width & $\mathrm{mm}$ & 10 & 10 \\
$W_{p m}$ & PM circumferential width & $\mathrm{deg}$. & 12 & 14 \\
$H_{s y}$ & Height of stator yoke & $\mathrm{mm}$ & 31 & 31 \\
$H_{s p}$ & Height of stator plate & $\mathrm{mm}$ & 8 & 9 \\
$H_{s t}$ & Height of tooth & $\mathrm{mm}$ & 14 & 15.7 \\
$H_{p m}$ & Height of PM & $\mathrm{mm}$ & 8 & 24 \\
$\rho$ & Core density & $\mathrm{g} / \mathrm{cm}{ }^{3}$ & 7.32 & 7.32 \\
$B_{r}$ & Remanence & $\mathrm{T}$ & 1.15 & 0.4 \\
$D$ & Diameter of copper wire & $\mathrm{mm}$ & 1.1 & 1.1 \\
$N$ & Turns of coil & - & 70 & 85 \\
\hline \hline
\end{tabular}

$$
\begin{aligned}
& \min : \mu_{f}(\mathrm{x}) \\
& \text { s.t. }\left\{\begin{array}{l}
\mu_{g_{i}}(\mathrm{x})+6 \sigma_{g_{i}}(\mathrm{x}) \leq 0, i=1, \ldots, 5 \\
x_{l j}+6 \sigma_{x_{j}} \leq \mu_{x_{j}} \leq x_{u j}-6 \sigma_{x_{j}}, j=1, \ldots, 16
\end{array}\right.
\end{aligned}
$$

To compare the product's reliability by using different design approaches, a criterion called as probability of failure (POF) has been used in many works. Assuming that all constraints in (5) or (6) are independent events, and then according to the Multiplication Theorem of Probability, the POF of the motor has the form as

$$
\mathrm{POF}=1-\prod_{i=1}^{m} P\left(g_{i} \leq 0\right) .
$$

For the robust optimization of (6), the multilevel optimization method shown in Section IV is employed. Taking the motor option of CPM with ferrite PMs under production option of small volume as an example, the main robust optimization steps are briefed as follows.

Step 1: Conduct local sensitivity analysis to obtain the sensitivity values for all parameters as shown in Fig. 5. Table VI lists the analysis data. As shown, $\left(\rho, L_{p m}, H_{s y}, L_{s p}, L_{s y}\right)$ are highly sensitive to the objective, so they are grouped into X1. Similarly, $\left(W_{p m}, H_{p m}, W_{s t}, L_{h d}, B_{r}\right)$ can be placed into group X2 based on their sensitivities. The others will be grouped into X3.

Step 2: Apply multilevel optimization method. The detailed optimization flowchart can be seen in Fig. 4. In each level, for example, X1, Kriging surrogate model will be constructed based on some FEM samples first. Then, the samples in MCA are randomly generated by assuming that each parameter follows a normal distribution with mean as the initial/optimal value and standard deviation as the $1 / 3$ of its manufacturing tolerance or the maximum material diversity. Finally, DEA is applied to optimize the obtained Kriging model. The $\mu$ and $\sigma$ values are estimated based on the Kriging model as well.

Step 3: Estimate the motor performance and POF based on the MCA analysis for the optimal results obtained in Step 2.

\section{E. Optimization results and comparison}

Tables VI to $\mathrm{X}$ list the optimization results for the three motor options in terms of two optimization approaches (deterministic and robust) and three production volumes, SV, MV and LV, respectively. In the tables, $R_{m}$ and $L_{m}$ stand for the outer radius and axial length of the optimized motor, which can determine the motor volume. The results can be compared in terms of the following three aspects.

(1) Sensitivity analysis data in terms of production volumes

Conventional method does not include production cost and conditions in the sensitivity analysis. In this work, it is found that the sensitivity values and order of the design parameters highly depend on the production conditions. As an example, Table VI lists the sensitivity order of design parameters in X1 (highly significant factor subspace) and X2 (significant factor subspace) for the CPM with ferrite PM. Compared with the conventional method, the sensitivity of each parameter (material or dimension) is not fixed but varies under different production volumes, because the production costs assigned to these design parameters are different.

(2) Comparison of performance and cost for all optimal motor designs and the initial designs from Tables VII-IX

Firstly, for the motor type, CPMs with both rare-earth and ferrite PMs are better than TFM as they have better performance (higher $P_{\text {out }}$ and $\eta$ ) and lower production cost.

Secondly, for the CPM, compared with ferrite PM CPM, the rare-earth CPM has higher power density (power/volume) but higher production cost. Thus, the ferrite PM CPM has the smallest production cost among these three options while the rare-earth PM CPM has the highest power density for all production volumes.

Thirdly, compared to the initial designs, the optimal designs for all motor options can provide better performance. For examples, Figs. 15 and 16 show the cost breakdowns of the ferrite CPM under three production volumes with initial and robust optimal designs, respectively. As shown, the cost has been decreased by $21.50 \%$ for large volume, $20.50 \%$ for medium volume and $20.84 \%$ for small volume productions, with an average of around $20 \%$. This is of great significance for industrial manufacturing and production.

(3) Comparison of sigma levels and POFs for deterministic and robust optimization approaches.

After optimization and MCA, the POFs and sigma levels for all constraints and motors can be obtained and compared. As an example, Table $\mathrm{X}$ lists the probability and sigma levels for all constraints and whole CPM with ferrite magnet with optimal designs from deterministic and robust approaches. As shown, the POFs of optimal deterministic design are higher than those of optimal robust designs. The sigma levels of some constraints are even less than 1 for deterministic approach. These are not acceptable in terms of industrial applications. There are several reasons for this high $\mathrm{POF}$, and two important ones are the temperature rise and output power. As shown, the first (output power) and last (temperature rise) constrains of deterministic approach are quite low, resulting in $40.78 \%$ POF for the motor in production. Figs. 17 and 18 show the distributions of temperature rise and output power for these two optimums, respectively. As shown, MCA samples (motor samples in production) of robust optimum meet all constraints, while deterministic optimum violates both constraints, i.e., the temperature rise exceeds the limit $75^{\circ} \mathrm{C}$ and the output power is lower than the specification of $675 \mathrm{~W}$. Therefore, robust design 
is very important for industry of electrical machines.

TABLE VI

SENSITIVITY ORDER OF PARAMETERS IN X1 AND X2 OF CPM WITH FERRITE

\begin{tabular}{lllllllllll}
\hline \hline Vol. & 1 & 2 & 3 & 4 & 5 & 6 & 7 & 8 & 9 & 10 \\
\hline SV & $\rho$ & $L_{p m}$ & $H_{s y}$ & $L_{s p}$ & $L_{s y}$ & $W_{p m}$ & $H_{p m}$ & $W_{s t}$ & $L_{h d}$ & $B_{r}$ \\
MV & $\rho$ & $L_{p m}$ & $W_{p m}$ & $H_{p m}$ & $L_{s p}$ & $L_{s y}$ & $H_{s y}$ & $D$ & $W_{s t}$ & $L_{h d}$ \\
LV & $L_{p m}$ & $\rho$ & $W_{p m}$ & $H_{p m}$ & $L_{s y}$ & $L_{s p}$ & $D$ & $H_{s y}$ & $W_{s t}$ & $L_{h d}$ \\
\hline \hline
\end{tabular}

TABLE VII

OPTIMIZATION RESULTS OF THE CPM WITH RARE EARTH PMS

\begin{tabular}{cccccccc}
\hline \hline \multirow{2}{*}{ Par. } & \multirow{3}{*}{ Unit } & \multicolumn{3}{c}{ Deterministic } & \multicolumn{3}{c}{ Robust } \\
& & SV & MV & LV & SV & MV & LV \\
\hline $\boldsymbol{\eta}$ & & $\mathbf{0 . 8 5 9}$ & $\mathbf{0 . 8 5 7}$ & $\mathbf{0 . 8 5 3}$ & $\mathbf{0 . 8 5 9}$ & $\mathbf{0 . 8 5 7}$ & $\mathbf{0 . 8 6 2}$ \\
Cost & AUD & 40 & 29.5 & 23.2 & 49.2 & 36.6 & 29.5 \\
$\boldsymbol{P}_{\text {out }}$ & $\boldsymbol{W}$ & $\mathbf{6 7 9}$ & $\mathbf{6 7 7}$ & $\mathbf{6 7 5}$ & $\mathbf{7 3 7}$ & $\mathbf{7 3 8}$ & $\mathbf{7 3 4}$ \\
$T_{\text {coil }}$ & ${ }^{\circ} \mathrm{C}$ & 74.40 & 74.75 & 74.50 & 72.1 & 72.5 & 72.7 \\
$\boldsymbol{R}_{\boldsymbol{m}}$ & $\boldsymbol{m} \boldsymbol{m}$ & $\mathbf{4 4 . 4 5}$ & $\mathbf{4 4 . 2}$ & $\mathbf{4 4 . 5 5}$ & $\mathbf{4 8 . 5 4}$ & $\mathbf{4 8 . 2 1}$ & $\mathbf{4 9 . 0}$ \\
$\boldsymbol{L}_{\boldsymbol{m}}$ & $\boldsymbol{m} \boldsymbol{m}$ & $\mathbf{8 9 . 2 2}$ & $\mathbf{8 8 . 9 5}$ & $\mathbf{9 2 . 2 2}$ & $\mathbf{8 9 . 9 4}$ & $\mathbf{9 2 . 4 6}$ & $\mathbf{9 0 . 6}$ \\
\hline \hline
\end{tabular}

TABLE VIII

OPTIMIZATION RESULTS OF THE CPM WITH FERRITE PM

\begin{tabular}{cccccccc}
\hline \hline \multirow{2}{*}{ Par. } & \multirow{3}{*}{ Unit } & \multicolumn{3}{c}{ Deterministic } & \multicolumn{3}{c}{ Robust } \\
& & SV & MV & LV & SV & MV & LV \\
\hline$\eta$ & & 0.850 & 0.858 & 0.851 & 0.854 & 0.847 & 0.854 \\
Cost & AUD & $\mathbf{3 4 . 6}$ & $\mathbf{2 3 . 2}$ & $\mathbf{1 7 . 6}$ & $\mathbf{3 7 . 2}$ & $\mathbf{2 6 . 9}$ & $\mathbf{1 9 . 5}$ \\
$P_{\text {out }}$ & $W$ & 679 & 675 & 675 & 718 & 712 & 713 \\
$T_{\text {coil }}$ & ${ }^{\circ} \mathrm{C}$ & 74.92 & 74.85 & 74.86 & 71.8 & 72.0 & 72.1 \\
$R_{m}$ & $m m$ & 49.05 & 49.76 & 49.64 & 53.54 & 53.75 & 52.26 \\
$L_{m}$ & $m m$ & 92.49 & 87.81 & 91.14 & 93.66 & 93.45 & 93.75 \\
\hline \hline
\end{tabular}

TABLE IX

OPTIMIZATION RESULTS OF TFM WITH RARE EARTH PMS

\begin{tabular}{cccccccc}
\hline \hline \multirow{2}{*}{ Par. } & \multirow{2}{*}{ Unit } & \multicolumn{3}{c}{ Deterministic } & \multicolumn{3}{c}{ Robust } \\
& & SV & MV & LV & SV & MV & LV \\
\hline$\eta$ & & 0.817 & 0.818 & 0.818 & 0.814 & 0.813 & 0.812 \\
Cost & AUD & 45.1 & 36.7 & 28.5 & 51.3 & 40.1 & 34 \\
$P_{\text {out }}$ & $W$ & 675 & 675 & 675 & 723 & 720 & 724 \\
$T_{\text {coil }}$ & ${ }^{\circ} \mathrm{C}$ & 74.2 & 74.1 & 73.9 & 72.1 & 72.5 & 72.7 \\
$R_{m}$ & $m m$ & 44.38 & 44.22 & 44.15 & 47.04 & 46.77 & 47.78 \\
$L_{m}$ & $m m$ & 88.55 & 88.77 & 88.77 & 90.12 & 91.29 & 92.64 \\
\hline \hline
\end{tabular}

TABLE $X$

RELIABILITY (p) AND RoBUST LEVEL ( $\sigma$ ) OF THE CPM IN LV CASE

\begin{tabular}{ccccc}
\hline \hline \multirow{2}{*}{ Constraint } & \multicolumn{2}{c}{ Deterministic } & \multicolumn{2}{c}{ Robust } \\
& $\mathrm{p}$ & $\sigma$ & $\mathrm{p}$ & $\sigma$ \\
\hline$g_{1}$ & 0.6596 & 0.95 & 1 & $>6$ \\
$g_{2}-g_{4}$ & 1 & $>6$ & 1 & $>6$ \\
$g_{5}$ & 0.8978 & 1.63 & 1 & $>6$ \\
Motor POF & \multicolumn{2}{c}{$40.78 \%$} & \multicolumn{2}{c}{$\sim 0.0 \%$} \\
\hline \hline
\end{tabular}

\section{VALIDATION OF OPTIMIZATION RESULTS}

Theoretically, it is a big challenge to validate the robust optimal results experimentally as the production environment should be developed, instead of laboratory prototype environment, for the mean, standard deviation and POF information. An alternative way is to validate all multi-physics analysis models and uncertainty data related to material diversity and manufacturing tolerances employed in the optimization. The optimal results should be reliable if all of these can be validated.

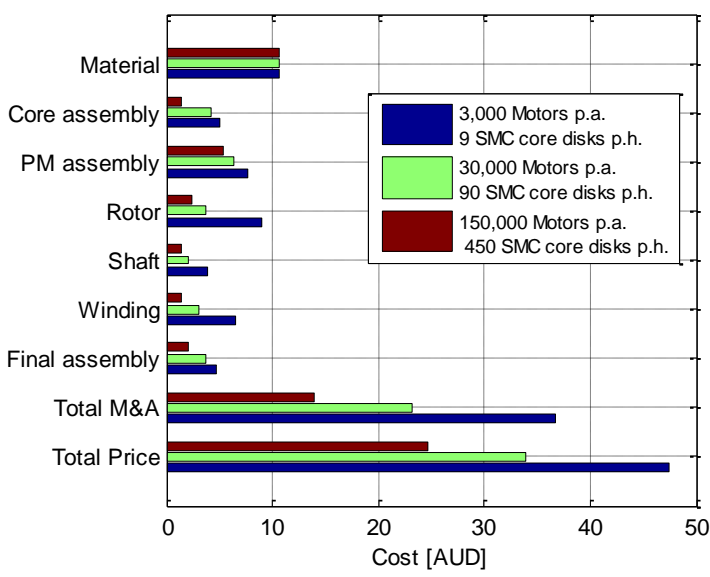

Fig. 15. Cost breakdown for ferrite PM CPM with initial design.

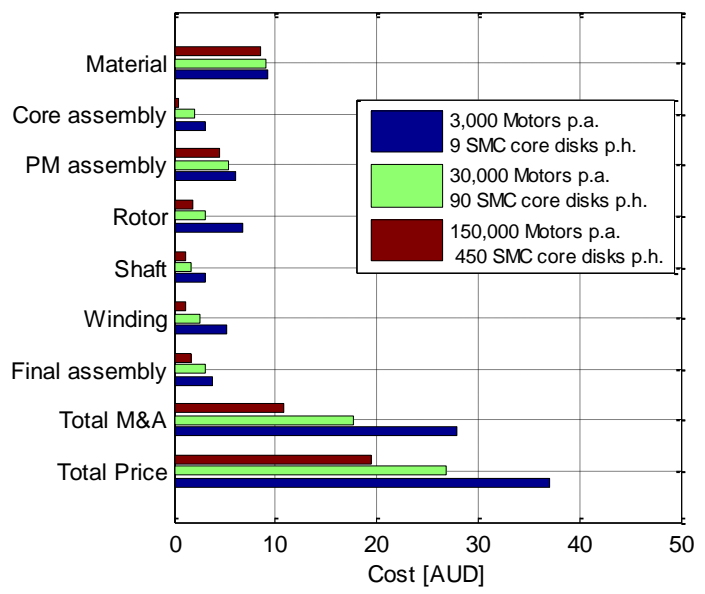

Fig. 16. Cost breakdown for ferrite PM CPM with robust optimal design.

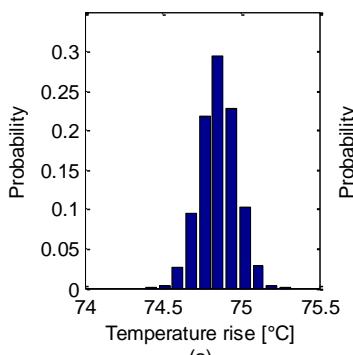

(a)

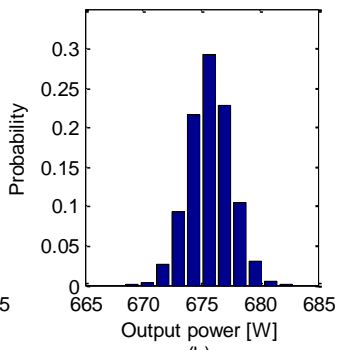

(b)

Fig. 17. Distributions for temperature rise and output power for ferrite PM CPM with deterministic optimum for large scale production.
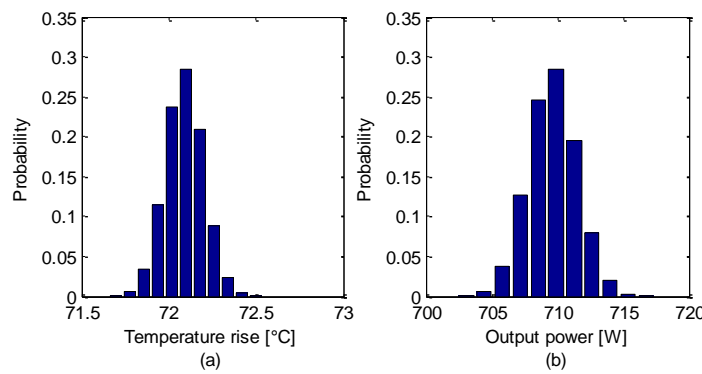

Fig. 18. Distributions for temperature rise and output power for ferrite PM CPM with robust optimum for large scale production. 

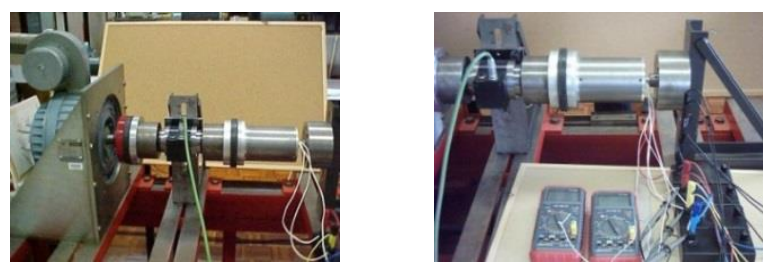

Fig. 19. Test platforms of a CPM (left) and a TFM (right) with SMC stators

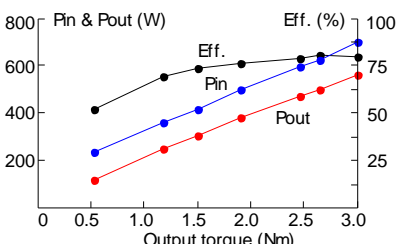

(a)

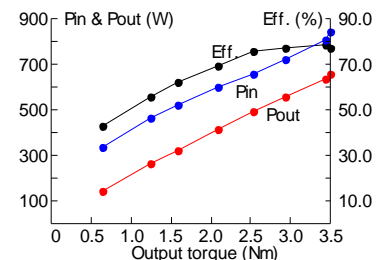

(b)
Fig. 20. Measured input, output powers and motor efficiency in terms of torques for (a) CPM, and (b) TFM for initial designs.

Firstly, regarding the multi-physics analysis model of the investigated PM-SMC motors, the validation can be conducted in terms of five aspects, namely back-EMF, cogging torque, core loss, inductance and thermal analysis.

Fig. 19 shows the two prototypes for CPM (rated $500 \mathrm{~W}$ ) and TFM (rated $640 \mathrm{~W}$ ) in experiments. Fig. 20 shows the measured efficiency, input and output powers for them. Figs. 21-23 show the comparisons of back EMF, cogging torque and core loss for calculated and experimental results. As shown, good alignments are observed for back-EMF and cogging torque curves. As the core loss is critical for the performance of SMC motors, more details are presented below.

For the accurate calculation of core loss in the PM-SMC motors, both alternating and rotational core losses should be included, due to the 3D nature of the magnetic fields in the PM-SMC motors. Fig. 23 (a) plots a 3D flux density locus (red) and its projections in different planes in a typical element in a stator tooth (point B in Fig. 7), showing that the flux density vector in the tooth is rotating elliptically in the $3 \mathrm{D}$ space. It is found that all the calculations are within 5\% error as shown in Fig. 23(b). For example, at the rated speed of $1800 \mathrm{rpm}$ (300 $\mathrm{Hz}$ ), the core loss for CPM prototype has been computed as $59.2 \mathrm{~W}$, which is very close to the measured value of $61.0 \mathrm{~W}$.

Secondly, Table XI compares several motor parameters, average flux and phase inductance (calculated by FEM), and temperature rise in winding (calculated by 3D thermal network model), with their measured values. Good alignments can be seen as well. Therefore, all models are of good accuracy and the optimization results should be reliable.

Thirdly, all the uncertainty data related to material diversity and manufacturing tolerances are obtained by experiments as well, such as the core density and PM characteristic data.

Therefore, all models and data used in the optimization have been validated by experiments. Consequently, the obtained optimization results should be reliable.

To have a further check of the optimal designs after optimization, Fig. 24 shows the back EMF and cogging torque curves for the optimal designs obtained from robust optimization in the case of large production volume of CPM with rare-earth $(\mathrm{NdFeB})$ and ferrite $\mathrm{PMs}$, respectively. As shown, good sine waves of EMF are observed and small cogging torque are obtained, particularly for the ferrite case. Finally, Fig. 24 shows the efficiency maps for those two optimal designed motors. As shown, the efficiency is relatively high for the region of rated speed at $1800 \mathrm{rev} / \mathrm{min}$.

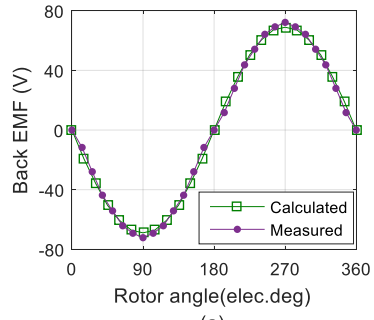

(a)

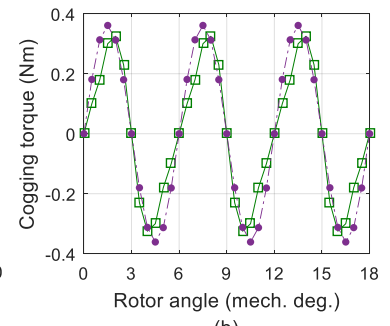

(b)
Fig. 21. Calculated and measured performances for the CPM, (a) back EMF, and (b) cogging torque.

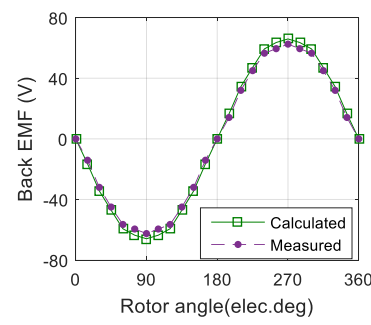

(a)

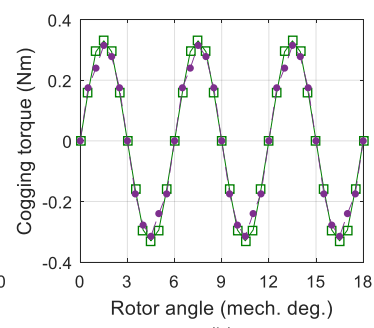

(b)
Fig. 22. Calculated and measured performances for the TFM, (a) back EMF, and (b) cogging torque.
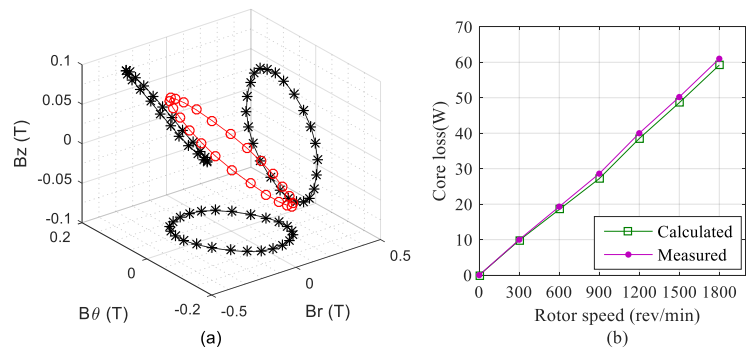

Fig. 23. 3D flux density locus and core loss data for the SMC CPM.

TABLE XI

Measured and CALCulated PARAmeters For CPM AND TFM

\begin{tabular}{cccccc}
\hline \hline \multirow{2}{*}{ Parameter } & \multirow{2}{*}{ Unit } & \multicolumn{2}{c}{ CPM } & \multicolumn{2}{c}{ TFM } \\
& & Cal. & Mea. & Cal. & Mea. \\
\hline Average flux & $\mathrm{mWb}$ & 0.487 & 0.488 & 0.280 & 0.272 \\
Phase inductance & $\mathrm{mH}$ & 5.35 & 5.78 & 6.68 & 6.53 \\
Temperature rise in coil & ${ }^{\circ} \mathrm{C}$ & 74 & 71 & 68 & 66 \\
\hline \hline
\end{tabular}

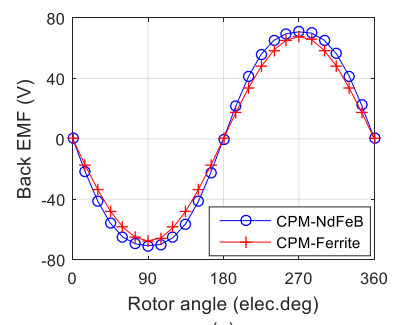

(a)

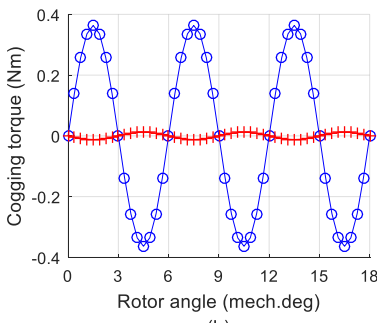

(b)
Fig. 24. (a) Cogging torque, and (b) back emf curves for the optimal CPM with Ferrite PM or NdFeB PM. 

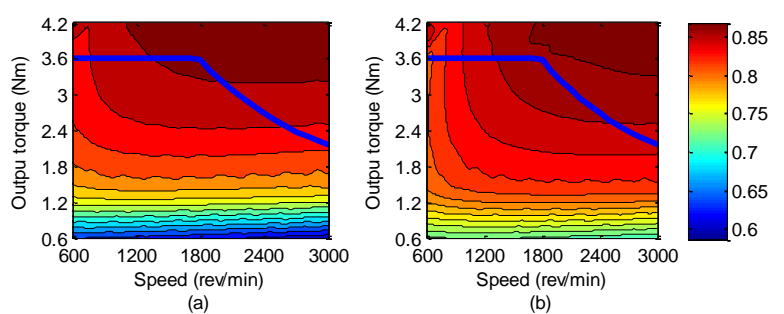

Fig. 25. Efficiency maps of CPMs with (a) NdFeB PM, (b) Ferrite PM.

\section{CONCLUSION}

An application-oriented robust design optimization method is presented for PM motors to improve both motor performance and manufacturing quality, and to reduce cost in the production environment in this work. A design example with PM-SMC motors is presented to show the effectiveness of the proposed method. In the implementation, three motor options with different materials and manufacturing methods are investigated under three production volumes. Then nine deterministic and nine robust optimizations (with 14-16 optimization parameters) are conducted separately to provide a fair comparison. Due to the huge computation cost, a multilevel robust optimization method is applied to improve the optimization efficiency. As shown, the optimal results vary in terms of motor type and production volume. The proposed method will benefit designers and manufacturers for achieving the optimal motor performance, high production reliability as well as minimal production cost. The proposed method can be applied to other kinds of electrical machines as well.

\section{REFERENCES}

[1] G. Lei, J. G. Zhu, and Y. G. Guo, Multidisciplinary Design Optimization Methods for Electrical Machines and Drive Systems, Springer, ISBN: 978-3-662-49269-7, 2016.

[2] J. A. Tapia, J. Pyrhönen, J. Puranen, P. Lindh, and S. Nyman, "Optimal design of large permanent magnet synchronous generators," IEEE Trans. Magn., vol. 49, no. 1, pp. 642-650, 2013.

[3] H. M. Hasanien, A. S. Abd-Rabou, and S. M. Sakr, "Design optimization of transverse flux linear motor for weight reduction and performance improvement using response surface model and genetic algorithm," IEEE Trans. Energy Convers., vol. 25, no. 3, pp. 598-605, Sep. 2010.

[4] D. Yao and D. M. Ionel, "A review of recent developments in electrical machine design optimization methods with a permanent magnet synchronous motor benchmark study," IEEE Trans. Ind. Appl., vol. 49, no. 3, pp. 1268-1275, Sep. 2013.

[5] P.-D. Pfister and Y. Perriard, "Very-high-speed slotless permanentmagnet motors: analytical modeling, optimization, design, and torque measurement methods," IEEE Trans. Ind. Electron., vol. 57, no. 1, pp. 296-303, Jan. 2010.

[6] K. I. Laskaris and A. G. Kladas, "Permanent-magnet shape optimization effects on synchronous motor performance," IEEE Trans. Ind. Electron., vol. 58, no. 9, pp. 3776-3783, Sep. 2011.

[7] K. Yamazaki and H. Ishigami, "Rotor-shape optimization of interior-permanent-magnet motors to reduce harmonic iron losses," IEEE Trans. Ind. Electron., vol. 57, no. 1, pp. 61-69, Jan. 2010.

[8] R. Nasiri-Zarandi, M. Mirsalim, and A. Cavagnino, "Analysis optimization and prototyping of a brushless DC limited-angle torque-motor with segmented rotor pole tip structure," IEEE Trans. Ind. Electron., vol. 62, no. 8, pp. 4985-4993, Aug. 2015.

[9] Z. Huang and J. Fang, "Multiphysics design and optimization of high-speed permanent-magnet electrical machines for air blower applications," IEEE Trans. Ind. Electron., vol. 63, pp. 2766-2774, 2016.

[10] I. Vese, F. Marignetti, and M. M. Radulescu, "Multiphysics approach to numerical modeling of a permanent-magnet tubular linear motor," IEEE Trans. Ind. Electron., vol. 57, no. 1, pp. 320-326, Jan. 2010.
[11] G. Bramerdorfer and A. C. Zavoianu, "Surrogate-based multi-objective optimization of electrical machine designs facilitating tolerance analysis," IEEE Trans. Magn., 2017, in press.

[12] I. Coenen, M. Giet, and K. Hameyer, "Manufacturing tolerances: Estimation and prediction of cogging torque influenced by magnetization faults," IEEE Trans. Magn., vol. 48, no. 5, pp. 1932-36, May 2012.

[13] M. A. Khan, I. Husain, M. R. Islam, and J. T. Klass, "Design of experiments to address manufacturing tolerances and process variations influencing cogging torque and back EMF in the mass production of the PMSMs," IEEE Trans. Ind. Appl., vol. 50, no. 1, pp. 346-355, 2014.

[14] A. J. Pina and L. Xu, "Analytical prediction of torque ripple in surface-mounted permanent magnet motors due to manufacturing variations," IEEE Trans. Energy Convers., vol. 31, pp. 1634-1644, 2016.

[15] G. Lei, T. Wang, J. Zhu, Y. Guo, and S. Wang, "System-level design optimization method for electrical drive systems-robust approach," IEEE Trans. Ind. Electron., vol. 62, no. 8, pp. 4702-4713, 2015.

[16] X. Ge and Z. Q. Zhu, "Influence of manufacturing tolerances on cogging torque in interior permanent magnet machines with eccentric and sinusoidal rotor contours," IEEE Trans Ind. Appl., 2017, in press.

[17] G. Lei, J. G. Zhu, C. C. Liu, and B. Ma, "Robust design optimization of electrical machines and drive systems for high quality mass production," in Proc. the 6th International Electric Drives Production Conference (EDPC), Nuremberg, Germany, 2016, pp. 217-223.

[18] A. Kampker, P. Burggräf, C. Nee, and M. K. Büning, "Integrated product and process development for electric engine production," in Proc. 18th Electric Power Distribution Conference, 2013, pp. 1-5.

[19] R. Cao, C. Mi, and M. Cheng, "Quantitative comparison of fluxswitching PM motors with IPM motor for EV, HEV and PHEV applications," IEEE Trans. Magn., vol. 48, no. 8, pp. 2374-84, 2012.

[20] J. T. Chen and Z. Q. Zhu, "Winding configurations and optimal stator and rotor pole combination of flux-switching PM brushless AC machines," IEEE Trans. Energy Convers., vol. 25, no. 2, pp. 293-302, 2010.

[21] J. T. Chen, Z. Q Zhu, S. Iwasaki, and R. P. Deodhar, "Influence of slot opening on optimal stator and rotor pole combination and electromagnetic performance of switched-flux PM brushless AC machines," IEEE Trans. Ind. Appl., vol. 47, no. 4, pp. 1681-1691, 2011.

[22] A. Krings, A. Boglietti, A. Cavagnino, and S. Sprague, "Soft magnetic material status and trends in electric machines," IEEE Trans. Ind. Electron, vol. 64, no. 3, pp. 2405-2414, 2017.

[23] Y. S. Kwon and W. J. Kim, "Electromagnetic analysis and steady-state performance of double-sided flat linear motor using soft magnetic composite," IEEE Trans. Ind. Electron, vol. 64, no. 3, pp. 2178-87, 2017.

[24] B. Zhang, T. Seidler, R. Dierken, and M. Doppelbauer, "Development of a yokeless and segmented armature axial flux machine," IEEE Trans. Ind. Electron., vol. 63, no. 4, pp. 2062-2071, 2016.

[25] J. Doering, G. Steinborn, and W. Hofmann, "Torque, power, losses, and heat calculation of a transverse flux reluctance machine with soft magnetic composite materials and disk-shaped rotor," IEEE Trans. Ind. Appl., vol. 51, pp. 1494-1504, 2015.

[26] T. Ishikawa, Y. Sato, and N. Kurita, "Performance of novel permanent magnet synchronous machines made of soft magnetic composite core," IEEE Trans. Magn., vol. 50, Art. 8105304, 2014.

[27] C. Liu, G. Lei, T. Wang, Y. Guo, Y. Wang, and J. Zhu, "Comparative study of small electrical machines with soft magnetic composite cores," IEEE Trans. Ind. Electron., vol. 64, no. 2, pp. 1049-1060, 2017.

[28] Y. Guo, J. G. Zhu, P. A. Watterson, and W. Wu, "Development of a PM transverse flux motor with soft magnetic composite core," IEEE Trans. Energy Convers., vol. 21, no. 2, pp. 426-434, 2006.

[29] J. G. Zhu, Y. G. Guo, Z. W. Lin, Y. J. Li, and Y. K. Huang, "Development of PM transverse flux motors with soft magnetic composite cores," IEEE Trans. Magn., vol. 47, no. 10, pp. 4376-4383, Oct. 2011.

[30] G. Lei, et al., "Multidisciplinary design analysis and optimization of a PM transverse flux machine with soft magnetic composite core," IEEE Trans. Magn., vol. 51, no. 11, Art. no. 8109704, Nov. 2015.

[31] G. Lei, C. Liu, J. Zhu, and Y. Guo, "Techniques for multilevel design optimization of permanent magnet motors," IEEE Trans. Energy Convers., vol. 30, no. 4, pp. 1574-1584, 2015.

[32] G. Lanza, J. Stoll, and A. Krämer, "Assessment of lamination stack production," in Proc. the 3rd International Electric Drives Production Conference (EDPC), Nuremberg, Germany, 2013, pp. 1-8.

[33] S. N. Lophaven, H. B. Nielsen, and J. Sondergaard, "DACE: A MATLAB Kriging toolbox version 2.0," Technical Report IMM-TR-2002-12, Technical University of Denmark, Copenhagen, 2002. 